two or three inches below its level. If, as is too often the case, it is prescribed for as chronic hepatitis, the constitution will be injured; for it is merely venous congestion, and the appropriate remedies are chalybeates and quinine.

Menstruation, if absent or deficient, will guide the physician when his patient is a female, and warn him against depletion: but, on the other hand, a frequent cause of headache is traceable to the debility arising from profuse menstruation and leucorrhoea. The profession are certainly indebted to Dr. Bennet for first pointing out to the practitioners of this country the intimate connexion there is between these symptoms and ulceration of the cervix uteri. But this ulceration or abrasion-for in many cases it is nothing more-must be curable by other means as well as by the application of nitrate of silver, as many unmarried females are restored to health by injections of acetate of zinc and the exhibition of sulphate of quinine accidulated. When the heart becomes flaccid from innutritious diet or over-exertion, there is not sufficient blood to spare for menstruation; and, on the other hand, profuse uterine secretions weaken equally the voluntary and involuntary muscles.

(To be continued.)

\section{ON THE ORIGIN AND CIRCULATION OF THE NERVOUS INFLUENCE.}

\section{BY JOHN M. STRACHAN, M.D.}

TuE question of the identity of the nervous influence with galvanic electricity has long been much disputed amongst physiologists, and much talent has been arranged on both sides. A great deal of the difficulty in which the question has been involved appears to have been caused by the want of a clear understanding of the source whence the influence is derived. This is a point which, although of great interest and importance has never been clearly established, and perhaps has never been properly investigated. Wishing to confine myself to this point, I shall, in the following remarks, take it for granted that the identity of the nervous influence with electricity is admitted.

The most prevalent opinion regarding the origin of the nervous influence is that it is generated in the brain and other nervous centres, and distributed through the body by the nerves. Before, however, we can be satisfied with this view, it is necessary to ascertain that the nervous centres can produce electricity, or, at least, that their capability of doing so is probable. But the very opposite of this is the case, there being scarcely a tissue of the animal system less likely to originate electricity than the substance of which the brain and spinal marrow consists.

I beg to suggest a source from which this electricity may be derived, which appears to have at least this advantage, that the electric fluid in sufficient quantity may be obtained from it,-namely, the chemical action which is constantly going on in the change of arterial into venous blood. Other chemical actions in the system may assist in producing the requisite amount of electricity; but, for the sake of simplicity, it may be as well to confine our attention at present to the one which is probably the most important.

In every chemical action galvanic electricity is evolved; a large amount must therefore be produced in the animal system by the change of arterial into venous blood. The following is a condensed view of what is now generally admitted to be the nature of the change referred to. The blood, after being exposed to the influence of the atmospheric air in the lungs, acquires a large amount of oxygen, so that the iron which it contains exists in the highest state of oxygenation as the peroxide. On reaching the capillaries, the peroxide of iron comes in contact with carbon, which attracts a portion of the oxygen; by this the peroxide is reduced to the state of protoxide of iron, and the carbon is converted into carbonic acid. The protoxide of iron and the carbonic acid, thus produced, immediately unite, forming the carbonate of iron.

It is quite elear that by these combinations galvanic elec tricity must be produced in great abundance; and as iron does not enter into the composition of any of the tissues or secretions of the body, it is probable that the production of electricity is the only purpose of this mineral existing in the blood in such quantity.

The chemical changes referred to are effected in the vessels situated between the termination of the arteries and the commencement of the reins. Now it is demonstrable that wherever arteries terminate and veins begin, there also are nerves dis. tributed, and as these consist of afferent and efferent fibrils, which have been proved to be conductors of electricity, we have only to suppose that the galvanism set free by the chemical action is taken up by the afferent nerves, and passing through the nervous centres, is returned by the efferent nerves to the same point, and thus a continued electric circuit is established, every modification of which must promote or retard the chemical change, and so influence the circulation of the blood; and, on the other hand, changes in the circulation of the blood must have a powerful influence in modifying the electric current. There is thus a constant circulation of nervous fluid dependent upon the circulation of the blood; and just as the heart does not generate, but only distributes the blood, so the brain and the other nervous centres do not produce, but serve to acceumulate, modify, and distribute the nervous influence.

This constant circulation of nervous fluid, in union with the circulation of the blood, is sufficient to give us an idea of organic life; and, perhaps, in some of the lower forms of animals this is all that exists. But as we rise in the soale of organization, the animal system becomes a much more complicated machine, having a variety of functions. It is therefore necessary to inquire how these may be performed.

The experiments of various inquirers have rendered it pro. bable that there are distinct nervous centres, and that to each of these belongs its own separate function. It is probable that these functions are performed, not as has generally been explained, by electric shocks communicated from or transmitted to the central organs, but by sustained and constant circuits of electricity produced by the changes of the blood in the capillaries, and by the modification of these currents by external or internal agencies.

On the supposition that the different nervous centres have separate functions, and that they all receive their electricity from the chemical changes of the blood, there comes the question whether each central organ has a completely distinet set of nerves, and that thus there are distinct nervous extremities, as well as distinct centres, for each function; or whether there is only one afferent and one efferent fibriI accompanying each capillary of the arteries and veins, which, receiving the electricity, conveys it to the nearest ganglion, from whence it is partly returned to the same point, thus maintaining the life of the part; but that a portion of the electricity is transmitted along other nerves to the more distant central organs, thus forming a longer circuit of motion or sensation.

It seems to be an universally received opinion that sensation results from impressions transmitted by the nerves to the central organ; that motion, on the other hand, arises from an influence sent from the central organ to the extremities of the nerves, thus conveying the idea that these centres are more sentient and more capible of originating motion than the other parts of the nervous system. This idea of impressions being conveyed to and from the brain is contrary to our conscionsness, and this should be a powerful reason for doubting its truth. When I prick my finger, I am not conscions of any impression being transmitted to the brain; on the contrary, I perceive the impression in the finger, and only there. Why, then, should we suppose that we are conscious only of impressions that are conveyed to the brain? It is true that any obstruction in the nerves connecting the external organs with the brain destroys sensation or motion. But this only shows that, for these functions to be performed, it is necessary that the electric circuit should be uninterrupted. The mind, however, is as likely to perceive any modification of that circuit at the point where it occurs, as at the distant central organ. And in the same way, motion may arise from some change effected at the extremities of the nerves, and not from an influence transmitted from the brain.

Regarding those operations which are considered as mental, I would merely hazard a conjecture-namely, that the large development of the cerebrum should not be considered as a central organ, but as a development of peripheral nerves, receiving electricity from the capillaries of the bloodvessels of the brain, and that these nerves have their centre in the commissures. This organ, which thus consists entirely of nerves and bloodvessels, may be, in accordance with general opinion, the seat of mental operations.

If it were established that there is a constant circulation of the nervous fluid, such as has been described, it would render our conceptions of the nerrons system as clear as those we possess of the circulation of the blood. The principle which governs it is the same as that of the galvanĩc battery, or a more apt illustration would be the electric telegraph, with its electricity conveyed by the positive and returned by the negar tire wire; and to carry out the comparison, the different 
nervous centres may be considered as so many telegraphic stations. There is this difference, however, that, instead of successive sparks of electricity, we have a sustained and continuous current, and consequently a provision in the circulation of the blood for keeping up a supply of the chemical ingredients necessary to maintain the stream.

If it were established that the circulation of nervous fluid is kept up in the manner described, it would improve our knowledge of the circulation of the blood, by showing a purpose effected by it even more important than the nourishment of the tissues; and we should then clearly understand the use of the oxygenation of the blood by respiration to be, the restoration to that fluid of the power of imparting electricity. Dollar, Clackmannanshire.

\section{Antèical Socítíts.}

ROYAL MEDICAL AND CHIRURGICAL SOCIETY.

TuESDaY, Feb. 14, 1854. - James Coprand, M.D., F.R.S., Prestdent.

Observations ON INJURIES OF THE HIP-JONNT, \&c. By Benjamin Travers, Esq., F.R. C.S., Junior.

(Communicated by his father.)

The author called to recollection that, in the twentieth volume of the Transactions of this Society, he had brought forward facts to prove that the head of the thigh-bone might be displaced, either directly upwards, or directly downwards; but he left it to be inferred concerning the latter form of injury that perhaps it did not occur withont fracture of some part of the os innominatum. His present object is to record the particulars of a well-marked ease of partial dislocation of the hip-joint, and also to point out the great probability of fissured acetabulum, unaccompanied by any other bony lesion, being a more common accident than has heretofore been supposed. He believes in the possibility of a "partial" dislocation of the femmr at the hip-joint in one direction-namely, towards the pubic margin of the articular cavity; the great trochanter being suddenly advanced as the head of the femur, restrained by the ligamentum teres, turns upon its axis. The injury is characterized by increase of width, and flattening of the buttock, and by alteration in the track of the hamstring muscles. The author then observes that the acetabulum may be cracked from injury, the head of the femur remaining entire. When the origin of the ligamentum teres is implicated this structure becomes absorbed and the head of the femur undergoes change in form and diminution in size. The subjects of this injury recover with certainty the power of locomotion, but with shortened limb. The shortening of the limb is progressive, being greater at six months than at two months, after which term it remains permanent. After the narration of several interesting cases the author concluded with remarks upon the mode of reducing dislocations, preferring the gentle management of muscles, to the old system of exhausting them by continued extension.

Mr. ERICHSEN would have felt more satisfied if Mr. Travers had adduced any instance of post-mortem examination of a case of fracture of the acetabulum. The first case of the supposed lesion brought forward by the author appeared to him (Mr. Erichsen) to resemble those instances of chronic arthritis which had been published by Mr. W. R. Smith, of Dublin, the injury received having perhaps ealled the chronic disease into a more active state. The only peculiar lesion advanced by Mr. Travers in favour of fracture of the acetabilum having existed was a furrow in that cavity; but this furrowing was sometimes observed in chronic disease of the joint, as the result of a tuberculated state of the upper part of the head of the femur. In the second case, which had been under treatment in the Charing-cross Fospital, the symptoms detailed closely resembled those of impacted fracture about the hip-joint. In the absence of any post-mortem examination it was difficul' to assent to the opinion of $\mathrm{M}_{r}$. Trwvers, that fracture of the acetabulum had existed independent of fracture of the pelvic bones.

Mr. LONSDALE had seen one or two cases of impacted fracture through the two trochanters, and agreed with Mir. Frichsen in thinking the second case was one of that character.

Mr. Moore remorked that the inpression he had derived from an observation of the drawings sent round was, that the appearances presented resembled those resulting from chronic infiammation. He did not think that the depression in the head of the bone was the result of a want of nutrition from rupture of the ligamentum teres, and, in the support of this opinion, referred to a case which had been recorded by himself in the Society's Transactions, in which the acetabulum had been fractured in three places, the head of the femur having been pushed through the cavity, and the ligamentum teres torn away; yet in this case, although after cleath no trace of the ligament existed, there was no loss of substance in the head of the bone.

Mr. B. TrAvERs, jun, , replied, and reiterated his opinion, that he had given a correct account of the nature of the accidents in the paper before the Society. He could not admit, after the experience he had had of fractures, that he had mistaken the cases detailed for fracture of the thigh-bone. There was no evidence in either of the cases of fracture of the head or neck of the femur. There might have been secondary deposits, as the result of inflammation in a rheumatic diathesis in the first case, but this he thought had nothing to do with the furrowed condition of the acetabulum. He considered that a crack might exist through the substance of the bones forming that cavity, without any displacement occurring, as was sometimes the case in the glenoid cavity. He still thought that in both cases he had correctly described the nature of the lesions which were present.

Notice of a Case of Skin Disease, accompanted With Partial Hypertrophy of the Mammary Gland. By James Alderson, M.D., F.R.S., Physician to St. Mary's Hospital, \&c.

THE subject of this case was a young lady, aged twenty, of fair complexion, light-blue eyes, and fair hair. When first seen, the left breast presented a diseased surface, at the upper part, to the extent of about four inches in length, by about an inch ard three-quarters in width. The appearances presented were, a perfectly smooth, polished surface, of an opaque, yellowish-white colour, like polished vellim or ivory; the margin of the diseased portion was defined by a stronglymarked border of injected vessels, but on the polished surface no vascularity could be perceived; there was no exudation whatever on any part of the breast; no crust nor scurf of any kind. This state of the skin had existed for nearly a twelve. month. The breast itself was larger than its fellow, and when handled presented several hard, resisting, nodulated tumours. A small, enlarged gland was situated in the left axilla, to which an absorbent vessel could be traced from the breast. No pain was experienced by the patient in the affected part. Mr. Hodgson was consulted on the case, and was led to pronounce an unfarourable prognosis, as it bore some resemblance to a case which ultimately displayed itself as carcinoma. Sir Benjamin Brodie subsequently was consulted, and in his large experience could only adduce a single similar case, in which the process of cure had been effected by throwing off successive layers of diseased skin, during which the extent of surface became continually reduced, the skin beneath ultimately assuming its natural aspect. He thought the disease less allied to carcinoma than to dry gangrene. The plan of treatment had hitherto been alkaline alteratives, with local application of iodine. The general treatment was but little varied: an alterative every other night, with the liquor potassa in liquid extract of sarsaparilla, and glycerine was directed to be rubbed on the part night and morning. After six months the surface of the breast had returned to its natural state, the patient stating that it faded gradually away. The author concluded with some remarks on the probable pathology of this peculiar clisease of the skin.

Mr. URE stated that he was one of the surgeons who had had the honour of being consulted respecting this interesting case. He could fully attest the fidelity of Dr. Alderson's account, and the aceuracy of the accompanying drawing. He considered the pecuitr parchment-like condition of the skin was dependent on some failure of nutrition, and had seemingly ori girated in connexion with äisorder of the catamenial function. Fe had suggested the local application of tincture of io line, and also the internal use of liguor potasse, with the vier of influencing the absorbent frnctions, and of sllaying crtaneous irritation. The patient, at the time he saw her, had several princtunte elevations of acne indurata upon the neck and shoulders. He had expressed a favourable opinion concerning the issue of the case, both as regarded the affection of the skin and the vartial hypertrophy of the mammary gland.

Mr. Fopasos was indeced to think that the disease under discussion was malignant, from having seen several cases very similar in anpeararce at first, wut which afterwards became carcinomatous, and destroyed the jatient. The peculiar rellumlike appearance of the skin in Dr. Alderson s case, existing 